\title{
Health differentials between citizens and immigrants in Europe: A heterogeneous convergence
}

\author{
Marwân-al-Qays Bousmah*, a, b, c, Jean-Baptiste Combes ${ }^{\dagger}$, d and \\ Mohammad Abu-Zaineh ${ }^{\ddagger}, \mathrm{c}$ \\ ${ }^{a}$ Aix Marseille Univ, INSERM, IRD, SESSTIM, Marseille, France \\ ${ }^{\mathrm{b}}$ ORS PACA, Observatoire régional de la santé Provence-Alpes-Côte d'Azur, Marseille, \\ France \\ ${ }^{c}$ Aix-Marseille Univ, CNRS, EHESS, Centrale Marseille, AMSE, and IDEP, Marseille, \\ France \\ ${ }^{\mathrm{d} U n i v}$ Rennes, EHESP, CNRS, ARENES - UMR 6051, F-35000 Rennes, France
}

\footnotetext{
*Corresponding author. SESSTIM, 19-21 boulevard Jean Moulin, 13305 Marseille, France. Tel.: +33491324600. E-mail address: marwan-al-qays.bousmah@univ-amu.fr

${ }^{\dagger}$ EHESP, 2 avenue Gaston Berger, 35000 Rennes, France. Tel.: +33299022718. E-mail address: jean-baptiste.combes@ehesp.fr

${ }_{\ddagger}^{\ddagger}$ AMSE, 5-9 Boulevard Bourdet, CS 50498, 13205 Marseille, France. Tel.: +33413552520. E-mail address: mohammad.abu-zaineh@inserm.fr
} 


\section{Acknowledgments}

This paper uses data from SHARE Waves 1, 2, 3 (SHARELIFE), 4 and 5 (DOIs: 10.6103/SHARE.w1.500, 10.6103/SHARE.w2.500, 10.6103/SHARE.w3.500, 10.6103/SHARE.w4.500, 10.6103/SHARE.w5.500), see Börsch-Supan et al. (2013) for methodological details. The SHARE data collection has been primarily funded by the European Commission through FP5 (QLK6-CT-2001-00360), FP6 (SHAREI3: RII-CT-2006-062193, COMPARE: CIT5-CT-2005-028857, SHARELIFE: CIT4CT-2006-028812) and FP7 (SHARE-PREP: №211909, SHARE-LEAP: №227822, SHARE M4: №261982). Additional funding from the German Ministry of Education and Research, the U.S. National Institute on Aging (U01_AG09740-13S2, P01_AG005842, P01_AG08291, P30_AG12815, R21_AG025169, Y1-AG-4553-01, IAG_BSR06-11, OGHA_04-064) and from various national funding sources is gratefully acknowledged (see www.share-project.org). This work has been carried out

thanks to the support of the A*MIDEX project (no. ANR-11-IDEX-0001-02) funded by the "Investissements d'Avenir" French Government program, managed by the French National Research Agency (ANR).

\section{Ethics approval}

Our study is based on data from SHARE Waves 1, 2, 3 (SHARELIFE), 4 and 5 (DOIs: 10.6103/SHARE.w1.500, 10.6103/SHARE.w2.500, 10.6103/SHARE.w3.500, 10.6103/SHARE.w4.500, 10.6103/SHARE.w5.500). The SHARE study is subject to continuous ethics review. During Waves 1 to 4, SHARE was reviewed and approved by the Ethics Committee of the University of Mannheim. Wave 4 of SHARE and the continuation of the project were reviewed and approved by the Ethics Council of the Max Planck Society. 


\section{Highlights:}

- We investigate differences in health patterns between immigrants and citizens in Europe.

- We use panel data on more than 100,000 older adults.

- Results show a reversal of health differentials between citizens and immigrants.

- There is also substantial heterogeneity across immigrant groups.

- The importance is stressed on the wealth of the country of origin. 
1

2

3

\title{
Health differentials between citizens and immigrants in Europe: A heterogeneous convergence
}

\begin{abstract}
The literature on immigration and health has provided mixed evidence on the health differentials between immigrants and citizens, while a growing body of evidence alludes to the unhealthy assimilation of immigrants. Relying on five different health measures, the present paper investigates the heterogeneity in health patterns between immigrants and citizens, and also between immigrants depending on their country of origin. We use panel data on more than 100,000 older adults living in nineteen European countries. Our panel data methodology allows for unobserved heterogeneity. We document the existence of a healthy immigrant effect, of an unhealthy convergence, and of a reversal of the health differentials between citizens and immigrants over time. We are able to estimate the time threshold after which immigrants' health becomes worse than that of citizens. We further document some heterogeneity in the convergence of health differentials between immigrants and citizens in Europe. Namely, the unhealthy convergence is more pronounced in terms of chronic conditions for immigrants from low-HDI countries, and in terms of self-assessed health and body-mass index for immigrants from medium- and high-HDI countries.
\end{abstract}

Keywords: Healthy immigrant effect $\cdot$ Europe $\cdot$ Health differentials $\cdot$ Health convergence $\cdot$ Immigration $\cdot$ Health economics.

JEL classification: F22 $\cdot$ I14 $\cdot$ J15. 


\section{Introduction}

The literature on immigration and health has provided mixed evidence on the health differentials between citizens and immigrants in developed countries. While some studies report that immigrants are in better health than citizens [1, 2, 3, 4, 5] providing support for the so-called healthy immigrant effect - others find the opposite [6, 7]. A growing body of evidence further shows that immigrants' health deteriorates with the length of residence in the host country [8, 9, 10, 11]. This phenomenon, sometimes called unhealthy assimilation, implies that the healthy immigrant effect, if any, is transitory. Describing and explaining the evolution of immigrants' health is of particular importance from a research and policy perspective. Once settled in the host country, immigrants would tend to have different health trajectories, not only relative to their native counterparts, but also among subgroups of immigrants.

The present paper attempts to assess whether the healthy immigrant effect, if any, is followed by a convergence of immigrants' health status toward that of citizens in Europe. We use panel data on more than 100,000 older adults living in nineteen European countries. The panel data methodology employed allows for unobserved heterogeneity. We document the existence of a healthy immigrant effect, of an unhealthy convergence, and of a reversal of the health differentials between citizens and immigrants over time. We are able to estimate the time threshold after which immigrant's health becomes worse than that of citizens. We further show that the effect on health of the immigrants' length of residence in the host country differs depending on the wealth of the country of origin and on the health measure considered. The present paper fits in the literature investigating the heterogeneity in health patterns

across immigrant groups, with a focus on the wealth of the immigrants' country of origin. Once settled in the European countries, immigrants from poor countries 
tend to work in the so-called 3D jobs (dirty, dangerous and demeaning) [12]. In this regard, the positive health selection (the healthy immigrant effect) would be higher for immigrants from poorer countries because of the physically demanding nature of their work [13]. This would also be a factor of faster health deterioration.

As we investigate the effect on health of the immigrants' length of stay in Europe, we focus on older immigrants. In North-West European countries, the three decades after 1945 were characterized by large-scale labour immigration. Immigration into

southern European destinations became significant from the 1970s [14]. We are now able to investigate the evolution of immigrants' health over a long time span, which explains the recent development of studies on older immigrants in Europe [14, 8, 9, 17, 15].

The remaining of the paper is organized as follows. Section 2 reviews the literature on immigrants' health in developed countries. The data and the econometric methodology are detailed in Sections 3 and 4, respectively. Section 5 presents the results, and Section 6 discusses the implications of the findings and concludes.

\section{Literature review: immigrant's health in developed countries}

In developed countries, health differentials between natives and immigrants and their evolution have been linked to the interplay of several factors. These include socio-economic conditions, individual factors (immigrants' behavior, health-related selection), and characteristics of both the country of origin (social norms, wealth) and of destination (social norms, integration policies towards immigrants). These health differentials are often mirrored by differences in access to health care.

McDonald and Kennedy [1] document a healthy immigrant effect and an unhealthy convergence in Canada regarding chronic conditions, but not self-assessed 
health, indicating that this phenomenon would be more pronounced in terms of physical health than health perceptions. Also in Canada, while showing the existence of a healthy immigrant effect in the case of depression, $\mathrm{Wu}$ and Schimmele [2] find that depression among immigrants increases with their length of stay.

In the United States, immigrants are less likely to have health insurance coverage and have lower health care use and spending than their native counterparts [16, 17, 18, 19, 20]. Observed differences in the access to health insurance and health care have been seen as one of the interplaying factors behind citizen-immigrant health differentials. Antecol and Bedard [3] show that immigrants have a lower bodymass index (BMI) upon arrival, but that they converge to that of the Americans as the length of stay increases. This relationship between BMI and duration of residence varies by gender and ethnicity [21], and by arrival cohorts, with more recent immigrant cohorts being more likely to be obese upon arrival and to experience a faster unhealthy convergence [22]. Other recent studies show that social norms in both the host country and the country of origin influence immigrants' health behaviors. Focusing on smoking behaviors, Leung [23] finds that assimilation in the United States is positively (negatively) associated with the likelihood of being a smoker for immigrants from low-smoking (high-smoking) countries.

Analyses of the relationship between immigrants' health and duration of residence in Europe have yielded mixed and sometimes conflicting evidence. Lindert et al. [24] report disparities across migrant groups in the prevalence of mental disorders and in the access and utilization of mental health services. Solé-Auró et al. [7] investigate the differences in health care utilization between older natives and immigrants. They find that older immigrants, particularly those who arrived recently, have a higher use of health care services (physician visits and hospital stays), due to differences in health, health behaviors, socio-economic status or countries' health system characteristics. 
Moullan and Jusot [25] show the existence of a North-South gradient in immigrants' health status, with immigrants in Italy and Spain having a better health status than in Belgium and France. Using three different health measures (self-reported health status, limiting long-standing illnesses, and self-reported chronic illness), Giannoni et al. [5] show that non-European immigrants tend to have better health in countries with pro-immigrant integration policies.

Using Swedish data, Pudaric et al. [26] show that, compared with Swedish-born individuals, country of birth is associated with poor health status (for immigrants from Southern Europe, Eastern Europe and Finland) and impaired instrumental activities of daily living (for Southern Europeans, Finns and refugees from developing countries). The authors also provide a theoretical framework for the link between immigrants' health deterioration and acculturation. They argue that poorly acculturated immigrants are exposed to long-term stress reactions due to a series of individual, structural and cultural factors, which may in turn harm their health. Bengtsson and Scott [27] highlight a gap in sickness benefit consumption between Swedish natives and immigrants, and also between immigrants depending on the country of birth. The authors show that while western immigrants and natives display similar patterns, immigrants from labor-sending countries, whose occupations are more physically demanding, have a higher average number of sick days.

In Spain, Malmusi et al. [8] document large migration-related health inequalities, with immigrants from poor areas being the worse-off group, which can be related to immigrants' socio-economic deprivation compared with natives. Evidence that immigrants' health deteriorates over time at a faster rate for immigrants working in high-risk jobs have been found in Spain [28] and in Germany [11].

In France, Vignier et al. [29] report that individuals in threat-related exile have a lower probability of accessing health care. Furthermore, it has been shown that 
undocumented immigrants experience great difficulty in exercising their rights to health care and tend to underutilize the State Medical Assistance [30]. The settlement process of recently arrived immigrants from sub-Saharan Africa has been shown to be long and precarious [31]. This long period of insecurity is mirrored by a lower health care utilization of recently arrived immigrants compared to the French-born population with equivalent health needs. This is reported in Berchet and Jusot [32], who also indicate that the healthy immigrant effect was mainly observed for immigrants who settled in France before the economic crisis of the mid-1970s. And even for these first- and second-generations immigrants, studies show that they suffer from premature dependency in old age [33], and, more generally, that their health became worse than that of the French-born population from the mid-2000s [34, 35]. Such health disparities are mainly due to differences in social capital, income and occupation status [36], and vary depending on the region of origin [37].

\section{Data}

We use data from the SHARE survey [38], which primarily aims at investigating ageing issues in Europe. The SHARE survey has also been used to investigate the relationship between immigration and health [9, 7, 15]. Our analysis is based on four waves conducted in 2004-2005, 2006-2007, 2010-2012 and 2013, respectively. These waves share the same methodology. The survey samples individuals over 50 years old in European countries. Individuals of the same household are also surveyed, regardless of their age. The 19 countries in the sample are Austria, Belgium, Czech Republic, Denmark, Estonia, France, Germany, Greece, Hungary, Ireland,

Italy, Luxembourg, the Netherlands, Poland, Portugal, Slovenia, Spain, Sweden, and Switzerland. 
Individuals' citizenship status (citizen or immigrant) is the main variable of interest. Among the alternative definitions of "immigrant", that are based on country of birth, nationality or length of stay in the host country [39], we have opted for the definition based on nationality, as the focus is on individuals who are legal residents, either permanent, temporary or refugees, and who represent a distinct category of the population. A study including three different categories - immigrants born foreigners in a foreign country, naturalized citizens and native citizens - would have been of great interest, but we cannot identify naturalized citizens in our data.

When individuals are surveyed for the first time, major life events are collected retrospectively. Thus, we know the immigrants' year of arrival, allowing us to compute the length of stay in the host country. As it is likely to be highly correlated with age, we divide the length of stay by the age. This measures the immigrants' share of lifetime in the host country. Immigrants in the sample arrived in the host country between 1916 and 2013. Immigrants' year of arrival distribution is shown in Figure 1.

\section{[Figure 1 about here]}

We also have information on the immigrants' country of origin, from which we construct a categorical variable based on the wealth of the country of origin. We use the UN's Human Development Index (HDI) as a measure of wealth. The HDI index doesn't measure economic growth alone but also considers individuals and their capabilities. GDP is a particular measure of wealth focusing only on this part of wealth which is measurable by a monetary value. By using HDI we intend to move beyond GDP and account a broader societal dimension of wealth and well-being. Based on their HDI value in 2015, countries of origin are classified as low human development $(\mathrm{HDI}<0.700)$, medium $(0.700>=\mathrm{HDI}<0.836)$ and high human 
development (HDI $>=0.836$ ). The 0.700 cut-off is commonly used to distinguish low- from medium-HDI countries, and the 0.836 cut-off represents the lowest HDI value among European Union countries (Hungary), as performed by Malmusi et al. [8]. Although immigrants in the sample arrived in the host country between 1916 and 2013, we only use the 2015 HDI of the country of origin, as more than $83 \%$ of the immigrants left their country of origin before the oldest year for which HDI data is available. The wealth of the country of origin should be interpreted with an appropriate degree of caution related to the fact that, although HDI data exhibit a strong path-dependency, some countries may be categorized in a given HDI category which was not the same at the time of migration.

Detailed definitions of the variables used are given in Table1. Five different health measures are used as dependent variables: (1) SAH (whether the individual reports a less than very good health), as is common in the literature (see for instance Antecol and Bedard 3 or Malmusi et al. 8), (2) the body-mass index (whether the individual is overweight or obese), (3) chronic conditions (the number of chronic diseases), (4) mental health (whether the individual is depressed), and (5) physical limitations (whether the individual has one or more limitations with activities of daily living $[\mathrm{ADL}])$. An extended discussion on the relevance of the health measures considered is provided in Appendix A1 of the electronic supplementary material. Descriptive statistics of the variables used in the analyses are given in Table A1 of the electronic supplementary material. Individuals in the sample are aged 22 to 103. Citizens' and immigrants' age distributions are relatively similar, as shown in Table A2 of the electronic supplementary material. Table A3 of the electronic supplementary material displays the percentage of immigrants by region of origin. The majority of immigrants come from the European Union or other European countries $(48.09 \%$ and $30.48 \%$, respectively). Stateless persons represent $8.07 \%$ of the sample of immigrants, 
and immigrants coming from Africa represent 7.74\%. Immigrants from the Americas or Asia and Oceania represent slightly less than 3\%, respectively.

$$
\text { [Table } 1 \text { about here] }
$$

\section{Econometric methodology}

We use a Mundlak [40] panel probit model for all binary dependent variables (Equation 1), and a Mundlak [40] panel Poisson model for the discrete positive dependent variable, that is, the number of chronic diseases (Equation 2). The Mundlak [40] specification is a fixed effect for all time-varying variables and a random effect for all time-constant variables. With a usual fixed effect model we would not be able to estimate a parameter for time-constant variables. With a random specification we would impose a certain specification to time-varying and time-constant variables. The Mundlak specification allows us to take the best of the two specification by transforming a random effect statistical routine in a fixed effect model for the timevarying variables if and only if the intra individuals means of these variables are included [41]. For individual $i(i=1, \ldots, n)$ in survey year $t(t=1, \ldots, T)$ :

$$
P\left(y_{i t}=1 \mid x_{i t}\right)=\Phi\left(x_{i t} \beta+c_{i}\right)
$$

where $\Phi$ is the standard normal cumulative distribution function.

$$
P\left(Y_{i t}=y_{i t} \mid x_{i t}\right)=F\left(y_{i t}, x_{i t} \beta+c_{i}\right)
$$

where $F(x, z)=P(X=x)$. $X$ is Poisson distributed with mean $\exp (z)$.

Equation 1 and Equation 2 are estimated using maximum likelihood. In both models, $x_{i t}$ is the vector of explanatory variables (some of these variables are con- 
stant over time) and $c_{i}$ is the time-invariant unobservable. We include the withinindividual means of the time-varying regressors, $\bar{x}_{i}$, to allow for the possibility that the unobserved time-invariant individual effect and the regressors are correlated (see Wooldridge 42 for further methodological details):

$$
c_{i}=\bar{x}_{i} \theta+\nu_{i} \quad \text { and } \quad E\left(c_{i} \mid x_{i}\right)=\bar{x}_{i} \theta
$$

where $\bar{x}_{i}$ is the panel-level mean of $x_{i t}$, and $\nu_{i}$ is a time-invariant unobservable that is uncorrelated to the regressors.

We include both age and period (survey wave) effects in the model. Cohort effects are not included due to the age-period-cohort identification problem and the impossibility to disentangle these three effects, as they are mathematically confounded [43]. Period effects are likely to capture unobserved factors common to all individuals in the sample. Country fixed effects are also included in the model to control for unobserved country-specific characteristics. Robust standard errors are computed at the household level to account for intra-household correlation. By using the Mundlak specification, the model accounts for the correlation between time-varying regressors and individual effects. We nonetheless acknowledge the potential presence of time-varying sources of endogeneity (for instance an income shock affecting both the access to health care and health outcomes).

Finally, we calculate average partial effects (APEs) to discuss the results in terms of magnitude. APEs are computed by averaging the partial effects across the population distribution of individual effects, which integrates out the unobserved heterogeneity [42].

As we focus on older immigrants, we cannot rule out the potential presence of a so-called salmon bias in our analysis. The literature on the salmon bias - immigrants 
who suffer from a long-term illness returning to their country of origin - has recently been reviewed in a study by Wallace and Kulu [44, reporting mixed evidence. More importantly, when there is evidence of a salmon bias, studies show that the mortality of those who return to their country of origin should have been unrealistically high in order to offset the migrant mortality advantage [45, 44]. From an econometric standpoint, the salmon bias would imply that there is measurement error in the dependent variable due to the fact that health of some immigrants would not be accounted for. The measurement error would thus be positively associated with the dummy variable for being an immigrant. In a similar manner, the immigrants' length of stay in the host country would be positively associated with the measurement error, in the sense that the parameter on length of stay would have been larger without the presence of a salmon bias, if any.

\section{Results}

\subsection{Baseline model}

Maximum likelihood estimates of the model (Equation 1 and Equation 2) and APEs are reported in Table A4 of the electronic supplementary material. The estimates show that immigrants are more likely to be in better health than citizens. This is true for all dependent variables except mental health, for which there is no significant difference between citizens and immigrants. The APE of being an immigrant ranges from -0.029 (for ADL limitations) to -0.067 (for chronic conditions). The probability of reporting poor $\mathrm{SAH}$ is $4.2 \%$-points lower for immigrants than for citizens. As reported in the introduction, such healthy immigrant effect has been highlighted previously. Nevertheless, the results reveal that the immigrants' length of stay - measured as the share of lifetime in the host country - is significantly and 
negatively associated with their health for all five health measures. A $10 \%$ increase in the share of lifetime in the host country increases the probability of reporting poor SAH by $1.13 \%$-points. Immigrants who spent most of their lives in the host country have a $11.3 \%$ higher probability of reporting poor health than those who arrived very recently. This is illustrated in Figure 2, which shows the estimated relationship between the immigrants' length of stay and their health for the five dependent variables.

[Figure 2 about here]

The estimated health of citizens is included for comparison. We can see that the healthy immigrant effect holds only for immigrants who arrived recently in the host country. For all five health measures, health worsens with the share of lifetime in the host country. Another important result is that, when the share of lifetime in the host country increases, immigrants' health eventually becomes poorer than that of citizens, as illustrated in Figure 2. Immigrants' self assessed and mental health reach the level of citizens when they have spent around 30 to $35 \%$ of their life in the host country (corresponding to, on average, approximately 18 to 21 years spent in the host country). For obesity and overweight, immigrants reach the level of citizens after having spent $45 \%$ of their life in the host country (on average approximately 27 years). This figure rises to $50 \%$ for chronic conditions (on average approximately 30 years) and $80 \%$ for ADL limitations (on average approximately 52 years).

We now interpret the coefficients for the other variables, which are common to all individuals in the sample. Compared to men, women are less likely to report poor health, to be overweight or obese, or to have physical limitations, but are more likely to have poor mental health. No significant gender difference is found for chronic conditions. Formal education is negatively associated with poor health 
status, with evidence of an education gradient. Current job situation is found to have different effects depending on the health measure considered. Note that the variable on current job situation has a specific category for permanently sick individuals, because their job situation is necessarily determined by their condition. This allows to appropriately capture the effect of being unemployed. In the case of $\mathrm{SAH}$ and mental health, being retired is associated with better health compared to all other categories (employed or self-employed, unemployed and homemaker or permanently sick). Being employed or self employed is associated with better health compared to all other categories in the case of BMI and chronic conditions. Regarding ADL limitations, being unemployed, homemaker or permanently sick is associated with poor health, while no significant association is found for the other categories. Marital status is found to have no effect on chronic conditions and ADL limitations. The likelihood of having poor mental health is $9 \%$-points higher for widowed than for individuals who are married or in a registered partnership (the reference category), while no significant association is found for the other categories (never married and divorced). Being divorced is positively associated with being overweight or obese. Never married and widowed individuals are more likely to have poor SAH. Drinking is positively associated with being overweight or obese, negatively associated with having poor mental health, and has no significant association with SAH, chronic conditions and ADL limitations. Physical inactivity increases the probability of having poor health for all health measures except BMI, for which it is negatively associated with being overweight or obese. Having children is associated with better mental and SAH, fewer chronic conditions, a higher likelihood of being overweight or obese and appears to be not associated with ADL limitations. Having grandchildren is associated with a higher likelihood of being overweight or obese, more chronic conditions, poor SAH (for three grandchildren or more) and is not associated with 
mental health and ADL limitations. For all health measures except BMI, the number of medical consultations is strongly associated with poor health, with a marked gradient. In the same way, the number of nights in hospital is linearly associated with poor health except for BMI, for which individuals who spent six or more nights in hospital in the year preceding the survey are less likely to be overweight or obese. Households' ability to make ends meet is associated with a higher likelihood of being overweight or obese. For all other health measures, the ability to make ends meet is associated with better health, with evidence of a socio-economic gradient in the case of self-assessed and mental health. The analysis of period (survey wave) effects indicates that the likelihood of being overweight or obese, of developing chronic diseases and of having poor mental health increased over time (from 2004-2005 to 2013). Almost no significant period effects are found for self-assessed health and ADL limitations. Finally, age is positively associated with poor health in the case of SAH and ADL limitations, and negatively associated with poor health in the case of BMI, chronic conditions and mental health. The unexpected negative effect of age for chronic conditions and mental health might due to the fact that, for an undocumented reason, the average number of chronic conditions and mental health are slightly decreasing between each individual's first and second interview (which may be distinct from the first and second wave, as some individuals entered the SHARE survey after the first survey-wave).

\subsection{Wealth of the country of origin}

To gain more understanding about the effects of being immigrant on health, we also investigate whether the effects highlighted previously change depending on the wealth of the country of origin. To do so, the immigrant status variable of the 
previous model is allowed to take on several values based on the level of human development of the country of origin. This variable is also interacted with the immigrants' share of lifetime in the host country. Maximum likelihood estimates and APEs are presented in Table A5 of the electronic supplementary material. Note that all the other results remain qualitatively the same, in terms of sign, magnitude, and statistical significance. Figure 3 shows the estimated relationship between the immigrants' length of stay and their probability of having poor health depending on the wealth of the country of origin. This is done for the five health measures.

\section{[Figure 3 about here]}

The results reveal that the effect of being an immigrant on health, and also that of the length of stay in the host country, varies depending on the wealth of the country of origin and the health measure considered. When arriving in the host country, immigrants from high HDI-countries are less likely to have poor SAH than citizens, while no significant association is found for the other categories. Nevertheless, when the share of lifetime in the host country increases, immigrants from medium- and high-HDI countries have poorer SAH, as illustrated in Figure 3a. The length of stay does not appear to change the SAH of stateless persons or immigrants from low-HDI countries. In the case of BMI, we found previously that immigrants are less likely to be overweight or obese than natives, but that this likelihood increases with the share of lifetime in the country. This result seems to hold mainly for immigrants from high-HDI countries. Although their likelihood of being overweight or obese is 10.3\%-points lower than that of citizens, immigrants from high-HDI countries who spent most of their lives in the host country are $21.4 \%$ more likely to be overweight or obese than those who arrived very recently, as shown in Figure $3 \mathrm{~b}$, The results regarding chronic conditions reveal a marked socio-economic gradient in the effect 
of being an immigrant and in that of their length of stay in the host country. Immigrants have a lower likelihood of developing chronic diseases, as shown previously. Compared to citizens, the likelihood of developing a chronic disease is $20.1 \%$-points lower for immigrants from low-HDI countries, while this figure falls to $8.4 \%$ and $3 \%$ for immigrants from medium- and high-HDI countries, respectively. We showed earlier that, among immigrant, the likelihood of developing chronic diseases increases with the share of lifetime in the country. We now show that, although immigrants from low-HDI countries have the lowest likelihood of having chronic conditions, they have the highest rate of health deterioration. There is a $28.2 \%$ difference between immigrants from low-HDI countries who arrived very recently and those who spent most of their lives in the host country. This figure falls to $14.4 \%$ and $7.7 \%$ for immigrants from medium- and high-HDI countries, respectively. The poorer the country of origin, the higher the deterioration of health with increasing share of lifetime in the host country. This is illustrated in Figure 3c. In the case of mental health, it seems that stateless persons have a lower likelihood of being depressed, while no significant association is found for the other groups. The results also indicate that immigrants from medium-HDI countries and stateless persons experience a mental health deterioration with the length of stay in the host country. Finally, the results show that the results found previously regarding ADL limitations hold mainly for immigrants from medium-HDI countries. They have a lower likelihood of having one or more ADL limitations, but this likelihood increases with the length of stay in the host country.

\section{Conclusion}

The question of whether there are significant health differentials between citizens 
and immigrants has extensively been addressed in the literature. Nonetheless, less is known about the evolution of immigrants' health over time. This paper attempted therefore to explore the dynamics of citizen-immigrant health differentials as well as the determinants of these dynamics. It employed panel models that allow for unobserved heterogeneity amongst more than 100,000 older persons living in nineteen European countries. Unlike previous studies that only focused on one or two measures of health, the present analysis has considered five different indicators of health status. This allowed to account for the multidimensionality of health and its evolution over time. Assessing the impact of the length of stay on immigrant health requires adjusting for the effect of age. The latter has been accounted for by using an age-adjusted measure of the length of stay in the host country.

Our paper highlighted large health differentials (1) between citizens and immigrants, (2) between immigrants depending on their length of stay in the host country, and (3) between immigrants depending on the wealth of their country of origin. These differentials are found to vary depending on the health measure considered. Some interesting results emerging from our analysis are worth discussing. First, our study generally, corroborates previous evidence on the healthy immigrant effect - according to which immigrants are initially more likely to be in better health as compared to citizens [1, 2, 3, 4, 5]. Secondly, our study also corroborates previous findings on the unhealthy assimilation of immigrants in developed countries [8, 9, 10, 11]. For all health measures considered, we found that, on average, immigrants' health deteriorates over time. In contrast to previous studies, our results not only shed light on the convergence of immigrants' health towards that of citizens. Interestingly, using an age-adjusted measure of the length of stay in the host country, we were able to show that this unhealthy convergence is such that the health of immigrants eventually becomes poorer than that of citizens. Thirdly, our results clearly revealed the 
presence of substantial heterogeneity in the observed unhealthy convergence, with respect to both the wealth of the country of origin and the health measured considered. For instance, in terms of chronic conditions, the health of immigrants from low-HDI countries tends to deteriorate faster than that of immigrants from richer countries. This is not surprising given that immigrants from low-HDI countries tend to work in the so-called 3D jobs (dirty, dangerous and demeaning) once settled in the European countries [12]. By contrast, the unhealthy convergence with respect to SAH seems to hold mainly for immigrants from medium- and high-HDI countries. In this case, the convergence of SAH towards that of citizens may well be a result of peer effects. Self-assessments of health have been shown to be influenced by social comparisons within age groups, especially among older people [46]. Thus, immigrants from medium- and high-HDI countries may tend to rate their health as similar to that of their native peers, which are more likely to belong to the same socio-economic group.

Altogether, such heterogeneity in the convergence of health differentials between immigrants and citizens in Europe seems to indicate that the unhealthy convergence is more pronounced in terms of chronic conditions for immigrants from low-HDI countries, and in terms of SAH and BMI for immigrants from medium- and highHDI countries. Future research shall attempt to identify the underlying factors of such heterogeneous convergence. 


\section{References}

[1] James Ted McDonald and Steven Kennedy. Insights into the 'healthy immigrant effect': health status and health service use of immigrants to Canada. Social Science $\mathscr{E}$ Medicine, 59(8):1613$1627,2004$.

[2] Zheng $\mathrm{Wu}$ and Christoph M Schimmele. The healthy migrant effect on depression: variation over time? Canadian Studies in Population, 32(2):271-295, 2005.

[3] Heather Antecol and Kelly Bedard. Unhealthy assimilation: why do immigrants converge to American health status levels? Demography, 43(2):337-360, 2006.

[4] Cristina Hernández-Quevedo and Dolores Jiménez-Rubio. A comparison of the health status and health care utilization patterns between foreigners and the national population in Spain: new evidence from the Spanish National Health Survey. Social Science $\mathscr{6}$ Medicine, 69(3): 370-378, 2009.

[5] Margherita Giannoni, Luisa Franzini, and Giuliano Masiero. Migrant integration policies and health inequalities in Europe. BMC Public Health, 16(1):463, 2016.

[6] Signe Smith Nielsen and Allan Krasnik. Poorer self-perceived health among migrants and ethnic minorities versus the majority population in Europe: a systematic review. International Journal of Public Health, 55(5):357-371, 2010.

[7] Aïda Solé-Auró, Montserrat Guillén, and Eileen M Crimmins. Health care usage among immigrants and native-born elderly populations in eleven European countries: results from SHARE. European Journal of Health Economics, 13(6):741-754, 2012.

[8] Davide Malmusi, Carme Borrell, and Joan Benach. Migration-related health inequalities: showing the complex interactions between gender, social class and place of origin. Social Science \& Medicine, 71(9):1610-1619, 2010.

[9] Donatella Lanari and Odoardo Bussini. International migration and health inequalities in later life. Ageing \&3 Society, 32(6):935-962, 2012.

[10] Santosh Jatrana, Samba Siva Rao Pasupuleti, and Ken Richardson. Nativity, duration of residence and chronic health conditions in Australia: Do trends converge towards the nativeborn population? Social Science $\mathcal{E}$ Medicine, 119:53-63, 2014.

[11] Osea Giuntella and Fabrizio Mazzonna. Do immigrants improve the health of natives? Journal of Health Economics, 43:140-153, 2015.

[12] Peter Stalker. The work of strangers: a survey of international labour migration. Geneva, Switzerland: International Labour Office, 1994.

[13] Yao Lu. Test of the "healthy migrant hypothesis": a longitudinal analysis of health selectivity of internal migration in Indonesia. Social Science \& Medicine, 67(8):1331-1339, 2008.

[14] Paul White. Migrant populations approaching old age: prospects in Europe. Journal of Ethnic and Migration Studies, 32(8):1283-1300, 2006.

[15] Amelie F Constant, Teresa García-Muñoz, Shoshana Neuman, and Tzahi Neuman. A "healthy immigrant effect" or a "sick immigrant effect"? Selection and policies matter. European Journal of Health Economics, 19(1):103-121, 2018.

[16] Olveen Carrasquillo, Angeles I Carrasquillo, and Steven Shea. Health insurance coverage of immigrants living in the United States: differences by citizenship status and country of origin. American Journal of Public Health, 90(6):917, 2000.

[17] Leighton $\mathrm{Ku}$ and Sheetal Matani. Left out: immigrants' access to health care and insurance. Health Affairs, 20(1):247-256, 2001. 
[18] Leighton $\mathrm{Ku}$. Health insurance coverage and medical expenditures of immigrants and nativeborn citizens in the United States. American Journal of Public Health, 99(7):1322-1328, 2009.

[19] Adriana M Reyes and Melissa Hardy. Another health insurance gap: gaining and losing coverage among natives and immigrants at older ages. Social Science Research, 43:145-156, 2014 .

[20] Wassim Tarraf, Elham Mahmoudi, Heather E Dillaway, and Hector M González. Health spending among working-age immigrants with disabilities compared to those born in the US. Disability and Health Journal, 9(3):479-490, 2016.

[21] Annie Ro and Georgiana Bostean. Duration of US stay and body mass index among Latino and Asian immigrants: a test of theoretical pathways. Social Science $\mathscr{6}$ Medicine, 144:39-47, 2015.

[22] Osea Giuntella and Luca Stella. The acceleration of immigrant unhealthy assimilation. Health Economics, 26(4):511-518, 2017.

[23] Leigh Ann Leung. Healthy and unhealthy assimilation: country of origin and smoking behavior among immigrants. Health Economics, 23(12):1411-1429, 2014.

[24] Jutta Lindert, Meryam Schouler-Ocak, A Heinz, and Stefan Priebe. Mental health, health care utilisation of migrants in Europe. European Psychiatry, 23:14-20, 2008.

[25] Yasser Moullan and Florence Jusot. Why is the 'healthy immigrant effect' different between European countries? European Journal of Public Health, 24(Supplement 1):80-86, 2014.

[26] Sonja Pudaric, Jan Sundquist, and Sven-Erik Johansson. Country of birth, instrumental activities of daily living, self-rated health and mortality: a Swedish population-based survey of people aged 55-74. Social Science E3 Medicine, 56(12):2493-2503, 2003.

[27] Tommy Bengtsson and Kirk Scott. Immigrant consumption of sickness benefits in Sweden, 1982-1991. The Journal of Socio-Economics, 35(3):440-457, 2006.

[28] Meritxell Solé, Luis Diaz-Serrano, and Marisol Rodríguez. Disparities in work, risk and health between immigrants and native-born Spaniards. Social Science $\mathcal{E} 3$ Medicine, 76:179-187, 2013.

[29] Nicolas Vignier, Pierre Chauvin, and Rosemary Dray-Spira. Un système de protection sociale universaliste, mais des barrières à l'accès aux soins encore trop nombreuses. In Annabel Desgrées du Loû and France Lert, editors, Parcours de vie et santé des Africains immigrés en France, pages 113-135. Paris, France: La Découverte, 2017.

[30] Jean-Marie André and Fabienne Azzedine. Access to healthcare for undocumented migrants in France: a critical examination of State Medical Assistance. Public Health Reviews, 37(1):5, 2016.

[31] A Gosselin, Annabel Desgrées du Loû, E Lelièvre, F Lert, R Dray-Spira, and N Lydié. How long do sub-Saharan migrants take to settle in France? Population et Sociétés, 533(May 2016), 2016.

[32] Caroline Berchet and Florence Jusot. État de santé et recours aux soins des immigrés en france: une revue de la littérature. Journal de Pédiatrie et de Puériculture, 25(2):120-125, 2012.

[33] Mathilde Plard, Aurélien Martineau, and Sébastien Fleuret. Les immigrés au seuil du grand âge. Hommes ${ }^{3}$ Migrations, (1):31-37, 2015.

[34] Claudine Attias-Donfut and Philippe Tessier. Santé et vieillissement des immigrés. Retraite et Société, (3):89-129, 2005.

[35] Florence Jusot, Jérôme Silva, Paul Dourgnon, and Catherine Sermet. Inégalités de santé liées 
à l'immigration en France. Revue Économique, 60(2):385-411, 2009.

[36] Caroline Berchet and Florence Jusot. Inégalités de santé liées à l'immigration et capital social: une analyse en décomposition. Économie Publique/Public Economics, 24-25:73-100, 2012.

[37] Nicolas Vaillant and François-Charles Wolff. Origin differences in self-reported health among older migrants living in France. Public Health, 124(2):90-98, 2010.

[38] Axel Börsch-Supan, Martina Brandt, Christian Hunkler, Thorsten Kneip, Julie Korbmacher, Frederic Malter, Barbara Schaan, Stephanie Stuck, and Sabrina Zuber. Data resource profile: the Survey of Health, Ageing and Retirement in Europe (SHARE). International Journal of Epidemiology, 42(4):992-1001, 2013.

[39] Bridget Anderson and Scott Blinder. Who counts as a migrant? Definitions and their consequences. Briefing, The Migration Observatory at the University of Oxford, http://www.migrationobservatory.ox.ac.uk/resources/briefings/who-counts-as-amigrant-definitions-and-their-consequences/, 2017.

[40] Yair Mundlak. On the pooling of time series and cross section data. Econometrica, 46(1): 69-85, 1978.

[41] Jean-Baptiste Combes, Ulf-Göran Gerdtham, and Johan Jarl. Equalisation of alcohol participation among socioeconomic groups over time: an analysis based on the total differential approach and longitudinal data from Sweden. International Journal for Equity in Health, 10 (1):10, 2011.

[42] Jeffrey M Wooldridge. Econometric analysis of cross section and panel data. Cambridge, MA: MIT Press, 2002.

[43] Andrew Bell and Kelvyn Jones. The impossibility of separating age, period and cohort effects. Social Science 83 Medicine, 93:163-165, 2013.

[44] Matthew Wallace and Hill Kulu. Can the salmon bias effect explain the migrant mortality advantage in England and Wales? Population, Space and Place, e2146, 2018.

[45] Hadewijch Vandenheede, Didier Willaert, Hannelore De Grande, Steven Simoens, and Christophe Vanroelen. Mortality in adult immigrants in the 2000s in Belgium: a test of the "healthy-migrant" and the "migration-as-rapid-health-transition" hypotheses. Tropical Medicine 85 International Health, 20(12):1832-1845, 2015.

[46] Marja Jylhä. What is self-rated health and why does it predict mortality? Towards a unified conceptual model. Social Science \& Medicine, 69(3):307-316, 2009. 
Table 1: Definitions of the dependent and independent variables

\begin{tabular}{|c|c|c|}
\hline Variable & Type & Definition \\
\hline \multicolumn{3}{|l|}{ Health measures (dependent variables) } \\
\hline Self-assessed health & Binary & 1 if the individual $i$ reports a less than very good health in survey wave $t ; 0$ otherwise \\
\hline BMI & Binary & 1 if the individual $i$ is overweight or obese in survey wave $t ; 0$ otherwise \\
\hline Chronic conditions & Discrete & Individual $i$ 's number of chronic diseases in survey wave $t$ \\
\hline Mental health & Binary & 1 if the individual $i$ is depressed in survey wave $t ; 0$ otherwise \\
\hline ADL limitations & Binary & 1 if the individual $i$ has one or more limitations with activities of daily living in survey wave $t ; 0$ otherwise \\
\hline Citizenship status & Binary & Citizen (=base category); Immigrant (legal residents, either permanent, temporary or refugee) \\
\hline Immigrant's share of lifetime in the host country & Continuous & Immigrant $i$ 's length of stay in the host country divided by his/her age \\
\hline Wealth of the country of origin & Categorical & Citizen (=base category); Low HDI $(<0.700)$; Medium HDI $(0.700>=$ HDI $<0.836)$; High HDI $(>=0.836)$; Stateless \\
\hline Gender & Binary & Male (=base category); Female \\
\hline Formal education & Categorical & None or Primary (=base category); Secondary; Tertiary \\
\hline Current job situation & Categorical & Retired (=base category); Employed or Self-employed; Unemployed; Homemaker or Permanently sick [in survey wave $t$ ] \\
\hline Marital status & Categorical & Married or Registered partnership (=base category); Never married; Divorced; Widowed [in survey wave $t$ ] \\
\hline Drinking & Binary & 1 if the individual $i$ was drinking more than 2 glasses of alcohol almost everyday in survey wave $t ; 0$ otherwise \\
\hline Physical inactivity & Binary & 1 if the individual $i$ was physically inactive in survey wave $t ; 0$ otherwise \\
\hline Number of children & Categorical & 0 (=base category); $1 ; 2 ; 3$ or more [in survey wave $t]$ \\
\hline Number of grandchildren & Categorical & 0 (=base category); $1 ; 2 ; 3$ or more [in survey wave $t]$ \\
\hline Doctor consultations & Categorical & Number of doctor consultations: 1st quartile (=base category); 2nd; 3rd; 4 th [in survey wave $t$ ] \\
\hline Nights in hospital & Categorical & 0 (=base category); Between 1 and $5 ; 6$ or more [in survey wave $t]$ \\
\hline Household able to make ends meet & Categorical & With great difficulty (=base category); With some difficulty; Fairly easily; Easily [in survey wave $t$ ] \\
\hline Country & Categorical & $\begin{array}{l}\text { Austria (=base category); Belgium; Czech Republic; Denmark; Estonia; France; Germany; Greece; Hungary; Ireland; } \\
\text { Italy; Luxembourg; the Netherlands; Poland; Portugal; Slovenia; Spain; Sweden; Switzerland }\end{array}$ \\
\hline Survey wave & Categorical & 2004-2005 (=base category); 2006-2007; 2010-2012; 2013 \\
\hline Age & Continuous & In years \\
\hline
\end{tabular}


Figure 1: Immigrants' year of arrival distribution

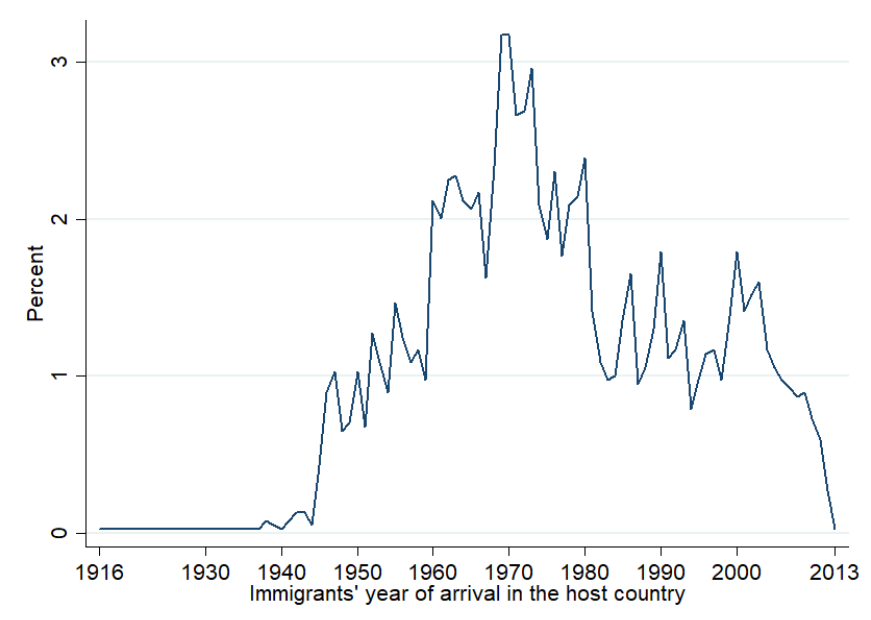


Figure 2: Effect of the immigrants' share of lifetime in the host country on health

(a) Self-assessed health

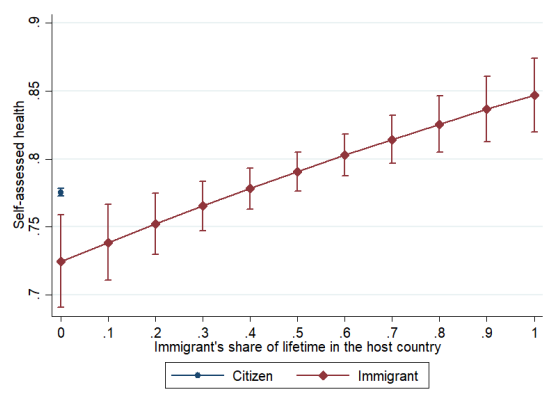

(b) BMI

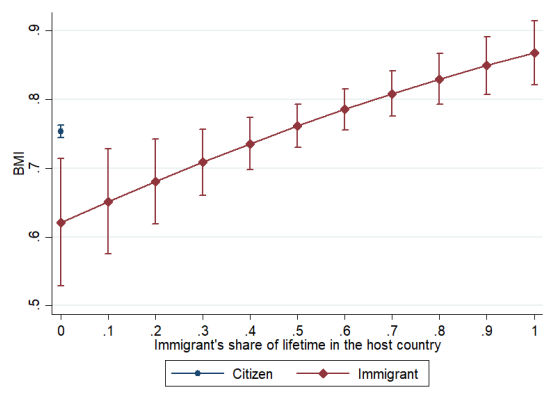

(c) Chronic conditions

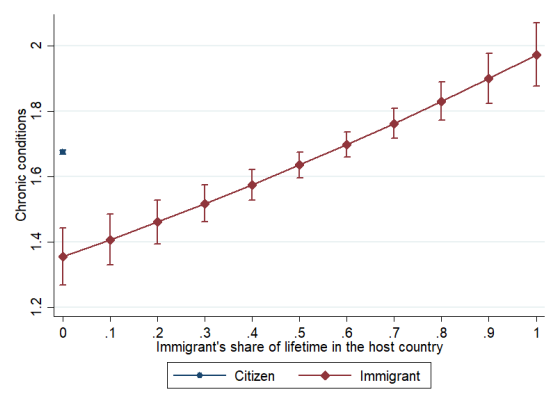

(d) Mental health



(e) ADL limitations

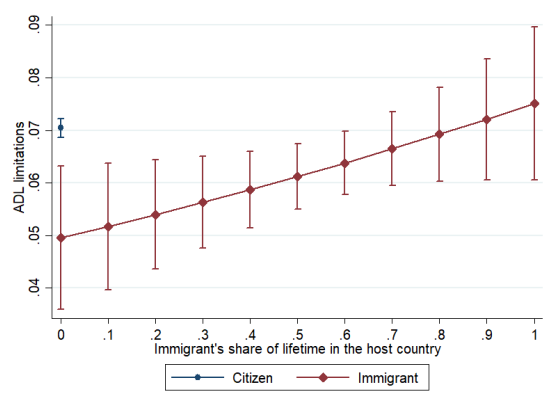


Figure 3: Effect of the immigrants' share of lifetime in the host country on health given the wealth of the country of origin

(a) Self-assessed health

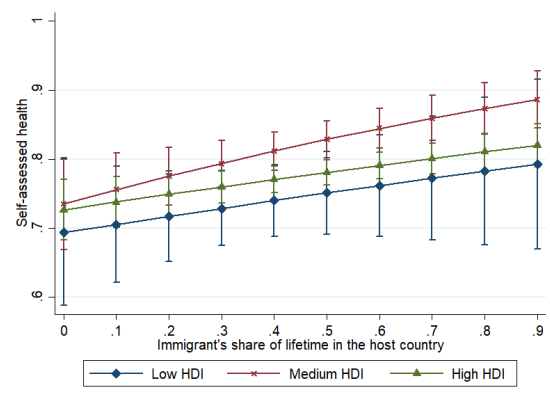

(b) BMI



(c) Chronic conditions

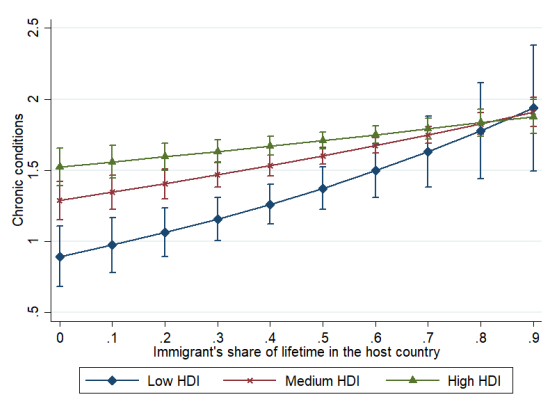

(d) Mental health

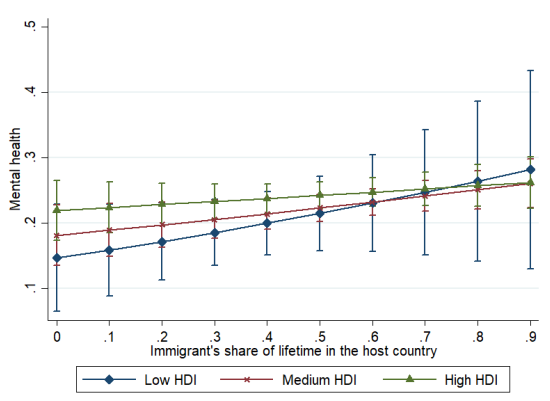

(e) ADL limitations

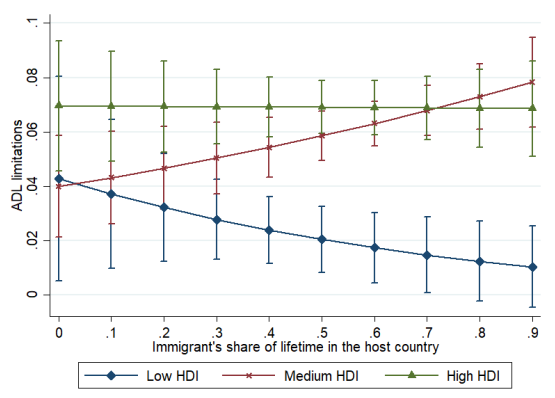

